\title{
Erratum
}

\section{Learning and Pattern Recognition in Spin Glass Models}

\author{
W. Kinzel \\ Institut für Festkörperforschung der Kernforschungsanlage Jülich, \\ Federal Republic of Germany
}

Z. Phys. B - Condensed Matter 60, 205-213 (1985)

Due to a programming error the numerical results were obtained from an alogrithm which is slightly different from the one described in Sect. 1. Consider a network with spins $\mathbf{S}=\left(S_{1}, S_{2}, \ldots, S_{N}\right), S_{i}= \pm 1$, and bonds $J_{i j}$. Its dynamics is defined by sequentially flipping the spins into their internal fields if necessary, i.e.,

$S_{i}^{\prime}=\operatorname{sgn} h_{i} ; \quad h_{i}=\sum_{j} J_{i j} S_{j}$

and updating the fields $h_{j}$ by

$h_{j}^{\prime}=h_{j}-\lambda J_{i j}\left(S_{i}-S_{i}^{\prime}\right) / 2$

where $\lambda$ is a parameter. With a given initial state $\mathbf{S}(0)$ and its internal fields

$h_{i}(0)=\sum_{j} J_{i j} S_{j}(0)$

this algorithm defines a deterministic time evolution $\mathbf{S}(t)$ in discrete times steps $t=0,1,2, \ldots$, where one time step is one sweep through the system. After time $t$ the internal fields are given by

$h_{i}(t)=(1-\lambda / 2) \sum_{j} J_{i j} S_{j}(0)+\lambda / 2 \sum_{j} J_{i j} S(t)$

For $\lambda=2$ one obtains the usual energy relaxation at zero temperature as discussed in Sect. II. For $\lambda=0$ the algorithm leads to a simultaneous spin flip which becomes stable after the first flip. In the article $\lambda=1$ was used. With Eq. (4) this leads to an algorithm which has some memory to the initial state.

Surprisingly, with $\lambda=1$ the retrieval error is less than the one of the energy relaxation $\lambda=2$. This is shown in Fig. 1, where the retrieval overlap is shown as a function of $\lambda$ for different noise levels $p_{n}$ of the initial patterns. The maximum is close to $\lambda=1$ for the case $N=400$.

Hence the numerical results for the retrieval overlap $q$ are somewhat smaller for the energy relaxation $\lambda$

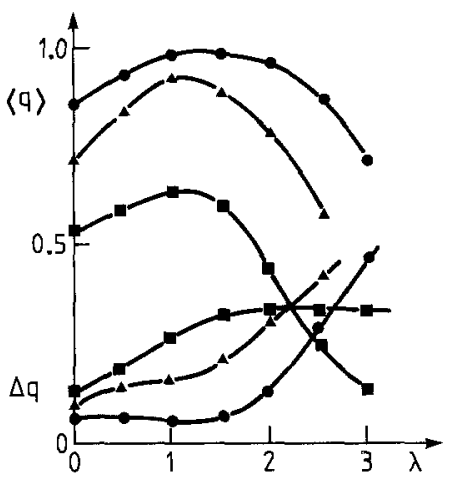

Fig. 1. Average retrieval overlap $\langle q\rangle$ and its standard deviation $\Delta q=\left\langle(q-\langle q\rangle)^{2}\right\rangle^{1 / 2}$ vs the parameter $\lambda$ of Eq. (2). The system size is $N=400$, and $M=30$ random patterns are stored with the Cooper relation, Eq. (7) of the article. The data are shown for different initial noise levels; dots: $p_{n}=0.3$, squares: $p_{n}=0.4$ and triangles: $p_{n}=0.35$.

$=2$ than the $q$ values of the article which used $\lambda=1$. In addition for $p_{n}=0$ and $p_{s} \gtrsim 0.15$ there is a strong size dependence of $\langle q\rangle$, and $P(q)$ is double-peaked in agreement with a discontinuous transition at $p_{s} \simeq 0.138$ which recently has been obtained analytically $[1]$.

I thank $\mathrm{H}$. Gutfreund for useful discussions and for pointing out to me the disagreement between the numerical results of the present paper and Ref. 1 .

\section{References}

1. Amit, D.J., Gutfreund, H., Sompolinsky, H.: Phys. Rev. Lett. $\mathbf{5 5}, 1530$ (1985); (to be published)

W. Kinzel

Institut für Festkörperforschung

Kernforschungsanlage Jülich $\mathrm{GmbH}$

Postfach 1913

D-5170 Jülich 1

Federal Republic of Germany 